\title{
Accurate OSNR Monitoring and Nonlinear Analysis of Signals in Dense WDM Systems Utilizing Polarization Interleaving of Adjacent Channels.
}

\author{
Petersen, Martin Nordal; Tokle, Torger
}

Published in:

European Conference on Optical Communications, 2006. ECOC 2006.

Link to article, DOI:

10.1109/ECOC.2006.4801304

Publication date:

2006

Document Version

Publisher's PDF, also known as Version of record

Link back to DTU Orbit

Citation (APA):

Petersen, M. N., \& Tokle, T. (2006). Accurate OSNR Monitoring and Nonlinear Analysis of Signals in Dense WDM Systems Utilizing Polarization Interleaving of Adjacent Channels. In European Conference on Optical Communications, 2006. ECOC 2006. (pp. 1-2). IEEE. https://doi.org/10.1109/ECOC.2006.4801304

\section{General rights}

Copyright and moral rights for the publications made accessible in the public portal are retained by the authors and/or other copyright owners and it is a condition of accessing publications that users recognise and abide by the legal requirements associated with these rights.

- Users may download and print one copy of any publication from the public portal for the purpose of private study or research.

- You may not further distribute the material or use it for any profit-making activity or commercial gain

- You may freely distribute the URL identifying the publication in the public portal 


\title{
Accurate OSNR Monitoring and Nonlinear Analysis of Signals in Dense WDM Systems Utilizing Polarization Interleaving of Adjacent Channels
}

\author{
Martin Nordal Petersen, Torger Tokle \\ COM·DTU, Department of Communications, Optics, and Materials, Technical University of \\ Denmark, Building 345V, DK-2800 Kgs. Lyngby, Denmark. \\ Email: mnp@com.dtu.dk
}

\begin{abstract}
A method for accurate OSNR monitoring and identification of nonlinear effects is experimentally demonstrated in dense WDM systems utilizing polarization interleaving of odd and even channels.
\end{abstract}

\section{Introduction}

In order to increase the cost and capacity further in optical communication systems, bit-rates and transmission distances are increasing while the channel spacing is decreasing. However, the increased spectral efficiency brings about problems such as linear crosstalk between channels, increased cross phase modulation (XPM) and four wave mixing (FWM) $[1,2]$. To reduce the detrimental impact of these effects in dense wavelength division multiplexing (WDM) systems, orthogonal polarization interleaving of adjacent channels has proven useful [2,3].

Another issue related to dense WDM systems is the evaluation of noise in the system. While coarse WDM systems offer the simple method of linear interpolation of the inter channel noise level to evaluate the optical signal to noise ratio (OSNR), dense WDM systems have overlapping channel side bands thus hiding the true noise level. Effective methods based on polarization extinction [4] as well as evaluation of degree of polarization (DOP) [5] have proven very effective in revealing the true noise level in dense WDM systems.

This work presents a method for accurate OSNR evaluations in dense WDM polarization interleaved systems. Based on the already used polarization interleaving equipment, the method is easily incorporated into these systems as few additions are needed. Furthermore, the method proved useful to evaluate system performance degradations due to self phase modulation (SPM), XPM and FWM. The experimental work was performed using a polarization interleaved $10 \mathrm{~Gb} / \mathrm{s}$ non-return-to-zero (NRZ) WDM system with $50 \mathrm{GHz}$ spacing.

\section{Operational Principle and Setup}

In dense WDM systems the channel sidebands will cover the noise level making it impossible to estimate an accurate OSNR. Using a polarization beam splitter (PBS), this method exploits the orthogonal polarized channels to separate odd and even channels and thus revealing the noise level within the inter channel band as illustrated in Fig. 1. The bottom right spectrum (OSA3) clearly reveals the noise level unlike the original spectrum shown on the left (OSA1). The spectrum shown as OSA2 in Fig. 1 resembles the second output of the PBS and is not used here. As will become evident from the results, an analysis of these spectra will also provide interesting information about the signal condition in terms of XPM and FWM.

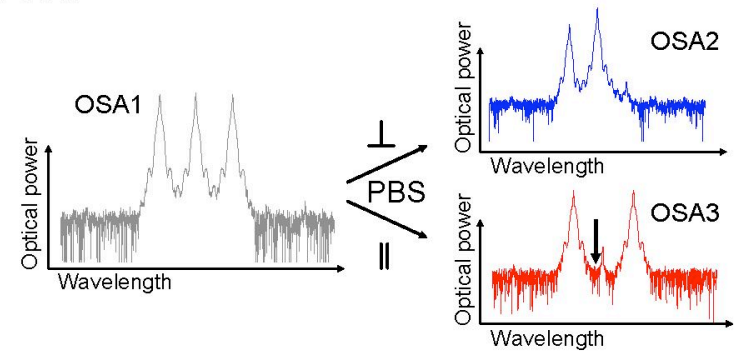

Fig. 1: The spectral representation before and after separation by the PBS. The bottom right spectrum now reveals the true noise level. The arrow in the OSA3 spectrum illustrates the later used "OSA3 power level".

Fig. 2 shows the setup used to demonstrate OSNR monitoring and the nonlinearity analysis. It shows the principle of polarization interleaving three $10 \mathrm{~Gb} / \mathrm{s}$ NRZ coded channels with a $50 \mathrm{GHz}$ spacing using a PBS. The signal from $T X 2$ is polarized orthogonal to the signal from $T X 1$ and $T X 3$. The WDM signal is amplified using an erbium doped fibre amplifier (EDFA) and transmitted through $80 \mathrm{~km}$ standard single mode fiber (SMF), which is $100 \%$ dispersion compensated using dispersion compensating fiber (DCF). A second EDFA and an attenuator act like an amplified spontaneous noise (ASE) source allowing to generate a signal with a variable OSNR. Nonlinearities can be introduced by increasing the power launched to the SMF.

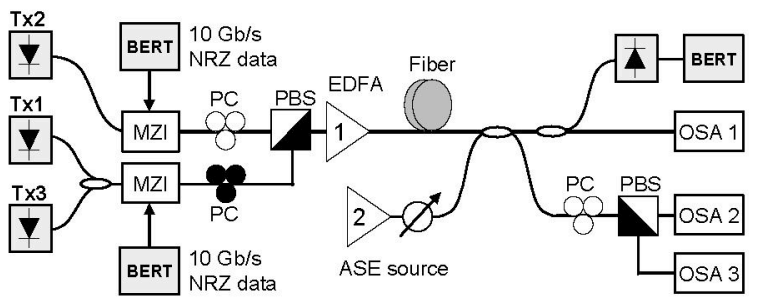

Fig. 2: Setup used to demonstrate accurate OSNR monitoring in polarization interleaved dense WDM systems.

After transmission the signal is split and one part continues to bit-error-rate (BER) estimation in the biterror-test-set (BERT). The other signal is separated using a PBS. The spectra are depicted in Fig. 1. 


\section{OSNR monitoring results}

Using the spectral information from OSA3 in Fig. 1 the OSNR can be evaluated. The noise level was varied and the OSNR was evaluated using the data from OSA3 (polarization filtered OSNR) as well as OSA1 (Traditional interpolation method). The result is shown in Fig. 3

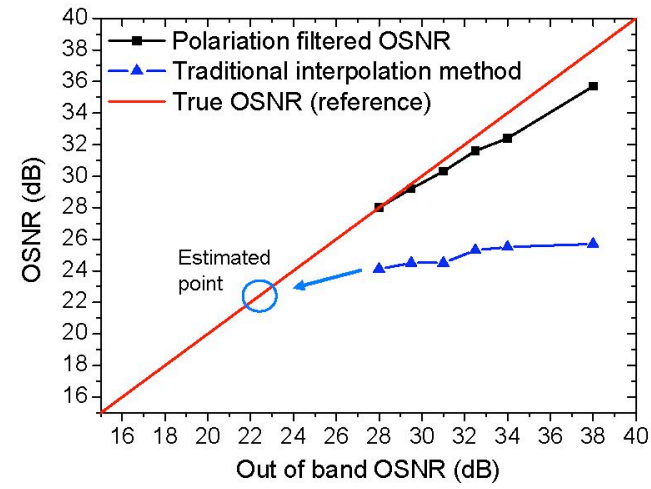

Fig. 3: Traditional OSNR evaluation method and the proposed method. The true OSNR is measured out-of-band.

The polarization filtered OSNR is found by taking the ratio of the carrier to the inter channel space illustrated by the arrow in the OSA3 spectrum in Fig. 1. Notice the improvement compared to the interpolation method. At very high OSNR values, however, the method becomes inaccurate due to spectral overlapping of channel 2 and 3 in the low power spectral region. However, as argued in [6], OSNR values above $25 \mathrm{~dB}$ are of little relevancy in $10 \mathrm{~Gb} / \mathrm{s}$ systems. Below $28 \mathrm{~dB}$ the OSNR measurements are fully accurate.

\section{XPM and FWM evaluation results}

Apart from being valuable in improving the accuracy of OSNR measurements, the technique can also provide valuable information about detrimental nonlinearities in the system. For this demonstration the same setup shown in Fig. 2 is used. Nonlinearities are excited by increasing the optical power to the SMF in increments up to $17 \mathrm{dBm}$.

For the purpose of the nonlinear analysis we now define an optical-signal-to-nonlinearity-ratio (OSNLR). The OSNLR is found as the ratio of the power in channel 1 to the OSA3 level defined in Fig. 1. The OSNLR will thus be influenced by SPM and XPM through spectral broadening but also by FWM and, to some extent, SBS which will both deplete the carrier. OSNLR and receiver sensitivity for channel 1 has been measured for different SMF input powers, as shown in Fig. 4. First of all, it is seen that the receiver sensitivity is evolving as expected. As the SMF input power increases, the nonlinear signal degradation will become significant, and the sensitivity is degraded.

In terms of the OSNLR curve, a rather good correlation with the receiver sensitivity is observed at input powers above $10 \mathrm{dBm}$. The increase in OSCR when going from low to higher input powers is expected as the probe channel power increases. Note the reverse OSNLR-axis. The drop in OSNLR at higher input powers can be explained through several nonlinear effects. First of all, the probe channel power will not continue to increase linearly with the input power as FWM and, to some extent SBS, will consume power from the probe channel. Furthermore, the measured OSA3 power will rise due to spectral broadening caused by SPM and XPM. The OSNLR plotted in Fig. 4 corresponds to the OSCR measurements excluding the carrier depleting effects of FWM and SBS and therefore this curve reveals the effect of spectral broadening caused by SPM and XPM only. The The OSNLRXTALK can be found as OSNLRXTALK $=$ OSNLR - $P_{\text {loss, }}$ where $P_{\text {loss }}$ is power loss in channel 1 due to FWM and SBS.

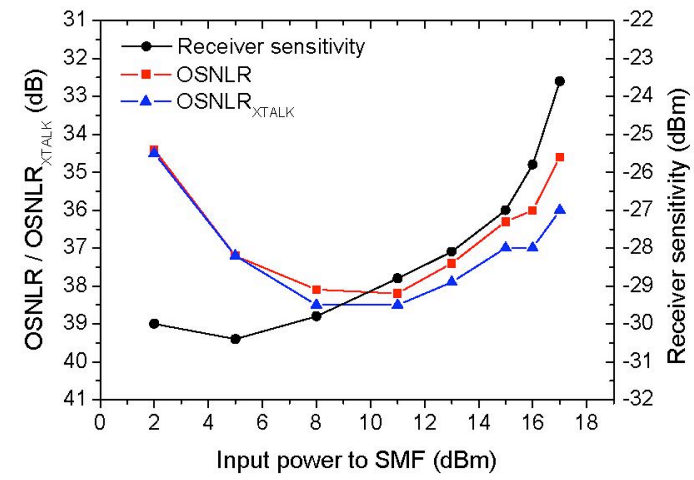

Fig. 4: The proposed method can successfully be used to evaluate the signal in terms of XPM and FWM.

The differences between the OSNLR and the

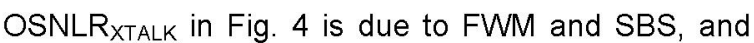
the drop in OSNLR XTALK at powers above $11 \mathrm{dBm}$ are thus identified to be due to SPM and XPM induced crosstalk. Fig. 4 therefore concludes that nonlinear effects influencing the signal quality can be successfully identified through the OSNLR. This explains the rather good correlation at high input powers with the receiver sensitivity observed from Fig. 4

\section{Conclusions}

This paper has presented a method for OSNR monitoring in dense WDM systems utilizing polarization interleaving. The method also proved useful to evaluate the impact of nonlinearities on the signal quality at high input powers. Contributions to signal degradations from SPM, XPM, FWM and SBS could be identified.

\section{References}

[1] K. Inoue, $P T L$, vol. 3, no. 6, pp. 560-563, 1991.

[2] T. Ono et al., Journ. of Quant. Elec., vol.34, no.11, 1998.

[3] D. Borne et al., PTL, vol.17, no.6, pp.1337-39, 2005

[4] M. Rasztovits-Wiech, ECOC'98, pp 549-550, 1998.

[5] M. Skold, OFC 2005, paper no. OThH3, 2005.

[6] D. C. Kilper, JLT., vol. 21, no. 5, pp. 1171-1178, 2003. 